\title{
Développer une intelligence commune du territoire
}

\section{Christine Partoune}

\section{(2) OpenEdition \\ Journals}

Édition électronique

URL : http://journals.openedition.org/ere/1059

DOI : $10.4000 /$ ere.1059

ISSN : 2561-2271

Éditeur

Centr'ERE

Référence électronique

Christine Partoune, "Développer une intelligence commune du territoire », Éducation relative à l'environnement [En ligne], Volume 10 | 2012, mis en ligne le 20 décembre 2012, consulté le 21 février 2020. URL : http://journals.openedition.org/ere/1059; DOI : 10.4000/ere.1059 


\title{
Développer une intelligence commune du territoire
}

\author{
Christine Partoune
}

1 À l'occasion d'une recherche-formation-action sur le développement et la gestion concertée des espaces publics (recherche dénommée TOPOZYM), l'équipe des formateurs-chercheurs de l'Institut d'Éco-pédagogie (IEP) a pu approfondir les fondements de l'éducation relative à l'environnement autour de la question singulière de l'éducation relative au territoire. Le projet de recherche-formation-action Topozym a été mené par l'Unité de Géographie Économique et Sociale de l'Université de Liège (UGES), l'Instituut voor Sociale en Economische Geografie de la Katholieke Universiteit Leuven (ISEG) et l'Institut d'Éco-pédagogie (IEP) entre janvier 2007 et janvier 2009. Le projet voulait contribuer à l'évolution des conceptions et des pratiques dans la gestion et l'usage des lieux et des espaces publics dans la perspective d'une «nouvelle gouvernance", où les citoyens jouent un rôle sans cesse croissant. Le but de la recherche était de produire un tableau de bord au service des personnes qui sont des vecteurs de changement de comportements des acteurs de l'espace (animateurs territoriaux, secteur éducatif, agents de développement local, etc.), pour les aider à concevoir et/ou à évaluer des démarches participatives efficientes et cohérentes dans le cadre de la gouvernance territoriale des lieux et des espaces publics. Le rapport final de cette recherche, en anglais, est disponible sur internet (De Graef et al., 2009), tandis que le tableau de bord n'existe qu'en français (Partoune et al., 2009).

2 La méthodologie de recherche reposait notamment sur la sélection de cinq cas contrastés d'espaces publics, en milieu rural ou urbain, en Flandres et en Wallonie, pour lesquels on pouvait espérer une amélioration dans la façon dont ils sont gérés ou aménagés grâce à une formation des acteurs impliqués. C'est l'IEP qui était chargé de la conception et de l'encadrement des formations, basées sur des méthodes participatives de type collaboratif, privilégiant la co-formation au sein de chaque groupe d'acteurs constitué pour chaque étude de cas.

3 L'identification des compétences à développer dans ce contexte a donné lieu à une proposition de travail originale : se saisir du cadre théorique posé par Howard Gardner 
(1997) à propos des multiples formes de l'intelligence pour focaliser l'analyse autour de ce qui nous apparaissait comme une forme particulière d'intelligence, l'intelligence du territoire (ou intelligence territoriale). Cette intelligence territoriale a rapidement été appréhendée sous l'angle des compétences collectives d'un groupe d'acteurs, c'est pourquoi nous parlerons désormais d'intelligence commune du territoire.

La question «À quoi reconnaît-on un groupe d'acteurs qui a développé une intelligence commune du territoire?» a été débattue régulièrement au sein du groupe interdisciplinaire des chercheurs associés, nourris par des lectures diverses; la réflexion a été enrichie sur la question de la mobilisation des acteurs au cours d'un workshop réunissant tous les participants aux groupes de travail des cinq études de cas, composés de citoyens, de fonctionnaires et d'experts dans des domaine très précis, ainsi que des membres du comité d'accompagnement de la recherche; un séminaire international réunissant une quarantaine de personnes impliquées à des degrés divers (scientifiques, bureaux d'étude, formateurs, animateurs socioculturels, fonctionnaires) dans des processus de participation citoyenne en matière d'espaces publics a permis d'approfondir la question des relations entre acteurs; la discussion des résultats de la recherche au cours du colloque Habiter (La Rochelle, août 2010) a permis de valider les propositions et d'encore les élargir ; enfin, c'est à partir de ce concept en construction d'intelligence commune du territoire que l'Institut d'Eco-pédagogie a défini un curriculum de formation en animation territoriale qui a été expérimenté en 2009-2010, donnant aux chercheurs l'occasion d'encore préciser et nuancer les choses.

Dans cet article, nous commencerons par préciser quelles visions du territoire nous choisissons et en quoi cela influence notre conception d'une éducation au territoire, en particulier si elle vise les espaces publics. Ensuite, nous exposerons quelle vision de l'intelligence territoriale oriente notre recherche et quels critères et indicateurs nous proposons pour reconnaître un groupe qui a développé une intelligence commune du territoire.

\section{L'espace public : un territoire à adopter}

6 Comme tout concept socialement construit, le mot "territoire" recouvre une multitude de significations. Il n'est pas utile pour notre propos de tenter de synthétiser les différentes façons de concevoir le territoire, mais de partir du principe qu'à tout le moins, il convient de tenir compte de la polysémie du concept, et surtout de ce que les différents sens donnés manifestent comme type de relation qui se noue avec le territoire.

7 Si nous nous référons aux discours dominants sur la gouvernance territoriale européenne, le territoire est surtout vu comme un espace économique utilisé pour satisfaire des besoins ou des aspirations: un espace que des personnes se sont approprié, de manière individuelle ou en groupe (groupes institutionnalisés ou non) pour l'habiter, l'exploiter, le baliser, échanger et communiquer à travers lui. Avec notre vision de l'éducation relative à l'environnement, nous y ajoutons d'autres dimensions : nous considérons aussi le territoire à la fois comme l'inscription manifeste de l'homme dans l'environnement et comme un espace existentiel marqué culturellement, dont les composantes et la structure jouent un rôle diffus mais déterminant sur une façon d'être au monde, à soi et aux autres, ainsi que sur la manière de se comporter dans l'espace et de se situer dans le temps. 
8 Alors, en miroir de l'éducation relative à l'environnement, l'éducation globale au territoire impliquerait une double mission: éduquer par le territoire et pour le territoire (tableau 1). À chacune de ces missions interconnectées correspondent différentes dimensions sociales de l'éducation, que nous proposons d'articuler en quatre axes.

Tableau 1 : Les dimensions sociales de l'éducation articulées en 4 axes

\begin{tabular}{|l|l|l|l|}
\hline & $\begin{array}{l}\text { Dimensions } \\
\text { sociales }\end{array}$ & Éducation centrée sur & Finalité \\
\hline \multirow{2}{*}{$\begin{array}{l}\text { Éducation par } \\
\text { le territoire }\end{array}$} & Humaniste & $\begin{array}{l}\text { La personne dans son/ses } \\
\text { territoire(s) }\end{array}$ & $\begin{array}{l}\text { Le développement de l'identité } \\
\text { territoriale }\end{array}$ \\
\cline { 2 - 5 } & Politique & $\begin{array}{l}\text { La société/les groupes } \\
\text { sociaux }\end{array}$ & $\begin{array}{l}\text { La mobilisation des citoyens } \\
\text { autour d'un projet de territoire } \\
\text { commun }\end{array}$ \\
\hline \multirow{2}{*}{$\begin{array}{l}\text { Éducation } \\
\text { pour } \\
\text { territoire }\end{array}$} & Épistémologique & $\begin{array}{l}\text { L'acquisition/la } \\
\text { construction } \\
\text { connaissances sur le } \\
\text { territoire }\end{array}$ & $\begin{array}{l}\text { La compréhension du } \\
\text { fonctionnement du territoire }\end{array}$ \\
\cline { 2 - 5 } & Praxique & $\begin{array}{l}\text { L'action sur le territoire } \\
\text { territoire }\end{array}$ \\
\hline
\end{tabular}

9 Axe 1, centré sur la personne : le territoire, un espace identitaire

10 L'éducation par le territoire, c'est d'abord une éducation centrée sur la personne qui vit ou qui utilise le territoire considéré, et qui a pour but de renforcer son ancrage territorial tout autant que ses qualités et compétences propres. Il s'agit par exemple d'apprendre à identifier les espaces qui sont devenus pour nous des territoires au sens éthologique du terme: de prendre conscience de nos attaches, de la façon dont nous avons habitons et avons marqué ces territoires, de ce que nous sommes prêts à y défendre, de l'importance des territoires que nous avons quittés (parfois à contrecœur ou de force).

11 En ce qui concerne ces portions particulières du territoire que sont les espaces publics, si l'on estime souhaitable qu'ils ne soient pas les espaces de personne, il faudrait qu'ils accèdent au statut de territoire au sens entendu supra, ce qui est rarement le cas. Pourtant, ce sont bien des territoires dans tous les sens du terme: lieux de cheminements plus ou moins réguliers, lieux de rendez-vous, lieux d'attente plus ou moins longue, lieux où s'exerce une activité particulière (promenade, achats au marché, manifestations, spectacle, sport, compétition), lieux de résidence pour les sans-logis. Il s'agit de reconnaître ces lieux-paysages publics qui constituent notre environnement quotidien et qui contribuent à nous apaiser ou à nous stresser, à nous réjouir ou à nous attrister, à nous faciliter la vie ou à la compliquer. Ce sont aussi des lieux d'ancrage subliminal pour tout un chacun d'une certaine vision du «bien commun » et de la notion de « service public ». 
12 La dimension de cet axe de l'éducation au territoire est profondément humaniste et existentielle : elle touche à notre histoire, nous permet d'y donner du sens et concerne notre identité profonde. Elle est marquée du sceau de notre culture, qui a conditionné notre manière d'explorer le territoire. Avons-nous eu la chance de l'explorer librement ou avons-nous toujours été tenus par la main pour le faire?

La manière dont nous avons été autorisés, depuis l'enfance, à parcourir des espaces de vie, joue probablement un rôle dans notre manière de penser. S'appuyant notamment sur les travaux Lakoff et Johnson (1985) qui considèrent que des structures chargées de sens issues de notre expérience incarnée principalement au niveau des mouvements de notre corps dans l'espace, notre manipulation d'objets et nos interactions perceptives (Fastrez, 1999) se constituent progressivement dans notre cerveau, John A. Waterworth (2002) suggère que le corps, avec sa relation au monde spatial réel ou virtuel qui l'entoure, est un lieu d'ancrage pour l'activité mentale ${ }^{1}$. Ces structures seraient incorporées sous la forme d'images-schémas qui traduiraient par exemple notre perception des notions de force, d'équilibre, de centre-périphérie, de partie-tout, de cycle, d'échelle. Elles organiseraient nos représentations mentales à un niveau plus général, plus abstrait que le niveau auquel nous formons ces images mentales. Ces images-schémas seraient structurées en "gestalt (groupement d'éléments dont le tout est plus que la somme des parties) et seraient articulées entre elles. Au fond, selon cette vision, notre façon de parcourir l'espace aurait une incidence sur notre façon de penser : notre manière d'investir progressivement des lieux, qui deviennent chargés de sens (chargés d'émotions, de mots, d'idées, de valeurs, etc.), et de relier ces lieux entre eux dans un schéma spatial mental dynamique, aurait une incidence sur notre manière de relier nos idées entre elles.

Autrement dit encore, nous pouvons nous demander dans quelle mesure notre manière de penser - plutôt linéaire ou systémique, plutôt intuitive ou rationnelle - est liée à la façon dont nous avons noué nos relations avec nos territoires de vie. Voilà déjà un des enjeux fondamentaux d'une éducation par le territoire.

\section{Axe 2, centré sur la société : le territoire, un espace à patrimonialiser}

Les hommes ne font pas qu'habiter tel ou tel lieu, ils y cohabitent (Lazzarotti, 2006). L'éducation par le territoire, c'est aussi une éducation centrée sur la société qui occupe ce territoire, et qui a pour but le changement social, voire sociétal. Cela veut dire non seulement décoder les enjeux et les rapports de force entre les acteurs, identifier les idéologies qui s'y affrontent et celles qui réussissent à structurer l'espace, mais aussi développer des «intelligences citoyennes» (Hansotte, 2005) qui s'exprimeront à travers l'émergence d'un projet commun ou d'une revendication collective, son analyse critique (qui/quoi bénéficie de quoi? au nom de qui/quoi? au détriment de qui/de quoi ?), la prise de parole dans l'espace public et la participation à des actions contribuant au développement du territoire.

En particulier, les espaces publics sont considérés ici comme la manifestation concrète d'une façon spécifique de voir le monde, comme une sorte de sceau culturel qui marque le territoire, comme une expression d'un savoir-vivre ensemble (Lazzarotti, 2006). Ils sont tout à la fois des lieux de rencontre, des lieux d'expression identitaire ou de mémoire collective, des lieux de loisirs ou de commerce, ou encore des lieux de représentation du pouvoir ou des artistes. 

décision prise par le groupe social qui détient le pouvoir, et qui le fait en référence à son univers culturel de référence, probablement de manière consciente et inconsciente. Cependant, cet espace public est susceptible d'être fréquenté par toute la population, qui ne partage pas forcément la même vision ou n'a pas les mêmes besoins, ce qui peut avoir des conséquences négatives (accidents, dégradation des lieux, détournement de l'usage prévu initialement, conflits d'usage, etc.) ; c'est toute la question des limites de la démocratie représentative. L'introduction de la concertation dans le développement et la gestion concertée des espaces publics est une façon pragmatique de tenir compte de la diversité culturelle dans notre société. développée dans une perspective critique, émancipatrice et démocratique. Il s'agit que l'environnement devienne un espace patrimonialisé. La notion de patrimoine fait ici référence à celle qui est définie par les tenants de la gestion patrimoniale: le patrimoine est constitué d'éléments matériels ou immatériels associés à un titulaire ou à un groupe de titulaires qui en sont le(s) porteur(s), qui cherchent à tout le moins à le maintenir et éventuellement à le développer "durablement » dans le temps et dans l'espace (Ollagnon, 2003, 2004 ; Stefanini et Vidal, 2001).

Pour Majo Hansotte et Hervé Brédif, le territoire en tant qu'espace patrimonialisé est un espace de projets communs, au service d'un «Pour Nous Tous» (Hansotte, 2005), «les différents membres de ce groupe ayant conscience de partager ensemble une communauté de destin et de projet en rapport avec cet espace et ses grandes qualités " (Brédif, 2004, p. 765). Il conviendrait en particulier de prendre conscience que les espaces publics représentent des territoires qui ne peuvent être intégralement gérés à l'échelle de la responsabilité d'individus, non plus qu'à l'échelle de la responsabilité de tel ou tel organe collectif.

L'orientation est (souvent) déjà fixée à partir d'un consensus imposé par le politique ou par les classes moyennes. Il est intéressant de se réunir, de regrouper les intérêts et de tenir compte de tous les intérêts dans les aménagements d'espaces publics et d'apporter un intérêt particulier aux groupes qui n'ont pas la parole. On constate souvent que cette question n'est pas posée et qu'elle implique un changement dans l'esprit des politiciens. (Storme, intervention orale lors du Séminaire international Topozym, 2008)

21 La dimension politique d'une éducation par le territoire implique donc des enjeux de «justice territoriale", d'accès pour tous aux espaces publics et aux chemins faire entendre sa voix concernant leur aménagement ou leur gestion. Et dans cette perspective se pose toute la question de l'apprentissage de la participation, dans et par l'action (voir axe 4).

\section{Axe 3, centré sur l'acquisition ou la production de connaissances nouvelles : le territoire,} un concept socialement construit.

L'éducation pour le territoire, c'est une éducation centrée sur le territoire en tant que tel, qui vise à mieux le connaître et le comprendre dans son fonctionnement écosociosystémique. Nous nous accordons à l'idée que tout savoir est socialement construit : d'une part, le social constitue la source du développement conceptuel d'une personne, qui apparaît à la fois comme le résultat de l'immersion de cette dernière dans un environnement culturel et comme le processus même de l'appropriation qu'il en fait ; d'autre part, le social est lié à l'activité de la personne qui apprend à s'approprier 
son environnement culturel, activité éminemment socialisée : ce n'est que dans la perspective d'utiliser ses savoirs à propos de l'environnement que la personne pourra élaborer leur signification culturelle, ce qui ne peut se faire indépendamment des relations interindividuelles qui en dessinent les contours culturels (Garnier et Sauvé, 1999).

Dès lors, l'analyse du territoire exige une clarification des concepts clés convoqués pour l'interpréter, des procédures d'investigation et des postulats de recherche. Le débat est convoqué pour construire les savoirs: les interactions sociales sont le lieu par excellence de la confrontation de nos représentations avec celles des autres, pouvant mener à des conflits sociocognitifs à forte dimension affective, et de la validation de leur signification.

On pourra, par exemple, essayer de comprendre comment et dans quel contexte le développement durable a émergé, comment certains acteurs le définissent et envisagent de l'appliquer au territoire, quels changements structurels sont apparus dans la société et quelles sont les critiques à cet égard. En particulier, la place de l'économie dans le schéma classique mérite d'être interrogée, tout comme la vision de l'environnement, principalement vu comme une contrainte, sauf s'il possède des ressources rentables ou qu'il peut rendre des «services écosystémiques». On interrogera aussi la notion même de "développement du territoire ». Et l'on encouragera l'éclairage multiple des concepts : qu'est-ce qui change dans notre vision du territoire si le terme développement signifie « une évolution positive qui se traduit le plus souvent par le maintien ou l'ajout de qualités", plutôt que "croissance économique »? «Évolution positive » donne une direction, mais pas le sens de celle-ci : elle peut s'envisager dans le sens de la croissance en termes de superficie ou d'usage, ou dans le sens d'une décroissance. Cette perspective reste ouverte et peut accueillir sans réserve des remises en cause de logiques de fonctionnement ou de valeurs aujourd'hui dominantes, comme la mobilité. La dimension de cet axe est épistémologique et socioconstructiviste. vance qui est promue. La vision traditionnelle, qui consiste à développer les compétences énoncées supra dans le chef des professionnels (gouvernance technocraticocentrée), est contrebalancée aujourd'hui par une vision plus démocratique, qui envisage complémentairement le développement de compétences collectives avec tous les acteurs, et singulièrement avec des citoyens (diagnostic partagé, coélaboration et cogestion de projets, etc.). Dans et par l'action commune, les acteurs réunis composent une communauté d'apprentissage qui contribue à la construction de nouvelles identités sociales et à la production de savoirs "sur mesure ", contextualisés; des savoirs signifiants, qui favorisent des changements d'attitude et le développement de nouvelles valeurs essentielles à l'encontre des tendances destructrices, qui fondent des relations d'un nouveau type avec le milieu de vie (Orellana, 2005). 
29 Attirons toutefois l'attention sur le fait qu'au commencement de chaque processus participatif, on part souvent du principe que les habitants/utilisateurs sont compétents pour réfléchir à propos du projet, alimenter le projet et prendre des décisions quant à leurs désirs, puis définir ensuite les grandes orientations et les principes fondamentaux du projet, c'est-à-dire son essence. Pourtant, les personnes réunies n'ont pas l'habitude de travailler ensemble. Quand c'est nécessaire, les gens doivent avoir la possibilité de rechercher, découvrir, approfondir, renforcer ensemble leurs compétences pour prendre part au projet. C'est là que réside la tâche importante de formation des participants, à travers une mission d'accompagnement: la formation est la clé de la participation! On sera attentif aux opportunités d'apprentissage quotidien, à l'apprentissage par et dans l'action et ce dans les divers contextes de vie.

Concernant les espaces publics, nous pourrions avancer que c'est au niveau de la perception de ces espaces en tant que «patrimoine » que tout se joue pour enclencher une mobilisation concrète, en relation avec l'ancrage sentimental dont ils sont potentiellement l'objet. Or, il y a tout lieu de penser que le sentiment d'appartenance à un espace public donné est très fluctuant, selon les lieux et les personnes, voire absent. Pour Henry Ollagnon,

Nous fonctionnons trop selon le principe : «Ce qui est à moi n'est pas à toi ». Or,

l'ambiance de la ville est quelque chose de public, issue des décisions individuelles.

Il faut donc réussir à imbriquer trois types de patrimoine : privé, public et commun.

Il y a alors « transappropriation ", mais celle-ci reste difficile à appréhender à cause

de notre fonctionnement par tiroir et non systémique. (Ollagnon, intervention

orale lors du Séminaire international Topozym, 2008)

31 Ce quatrième axe révèle à quel point le processus d'éducation par et pour le territoire est complexe. Partir de la sensibilisation par le territoire pour qu'il devienne patrimoine partagé, passer par la sensibilisation à la participation citoyenne et acquérir des compétences nouvelles en la matière, construire de nouveaux savoirs sur le territoire avec d'autres pour en saisir les enjeux et y développer des projets qui ont du sens : c'est un processus qui s'apparente à une véritable démarche d'adoption.

\section{L'intelligence commune du territoire : une nouvelle forme d'intelligence?}

La notion d'intelligence du territoire dont il est question ici s'écarte de la notion d'intelligence territoriale utilisée dans le champ de la gouvernance territoriale, bien qu'elle y soit apparentée. Cette dernière, bien que polysémique dans ses définitions, se réfère en général à une méthode de gestion économique du territoire reposant sur la mise en réseau des acteurs, afin de collecter et traiter les informations d'une manière plus pertinente. L'intelligence dont il est question pour notre propos est à prendre au sens de «capacité à résoudre des problèmes ou à produire des biens, de différentes natures, ayant une valeur dans un contexte culturel ou collectif précis » (Gardner, 1997).

33 Partant de cette définition générale, l'intelligence du territoire pourrait donc être définie comme la «capacité à résoudre des problèmes qui se posent sur un territoire donné ou à y développer des projets, d'une manière qui soit reconnue comme valable par les personnes concernées». Si, comme nous l'avons développé supra, nous considérons le territoire comme un espace investi et patrimonialisé par un groupe 
humain, il convient de ne pas réduire cette idée d'intelligence du territoire à un ensemble de compétences individuelles mais d'envisager d'emblée l'idée d'une intelligence commune du territoire, qui supposerait l'acquisition de compétences collectives. Une intelligence commune du territoire correspondrait à une manière d'y vivre et d'en vivre qui serait spécifiquement liée au fait de ressentir-penser-agircommuniquer en groupe, les membres du territoire considéré possédant des habiletés émergentes qu'aucun acteur ou groupe d'acteur n'aurait pu acquérir isolément.

\section{Critères et indicateurs pour repérer une intelligence commune du territoire}

La méthode de travail d'Howard Gardner et de ses associés chercheurs consiste à observer comment les personnes se comportent et à identifier les tâches qu'elles réussissent particulièrement bien, puis à détailler les indices permettant de reconnaître telle ou telle forme d'intelligence. Quels sont les indices pour reconnaître l'existence d'une intelligence commune du territoire, lorsque nous le parcourons et que nous entrons en contact avec ses habitants ou avec les personnes qui y exercent des activités ? Les propositions présentées dans l'encadré sont offertes à la discussion à travers cet article. L'ordre dans lequel les critères et indices sont présentés est fortuit mais il est sans doute souhaitable de baliser un travail en situation (par exemple s'il s'agit de définir un processus évolutif) en imaginant une progression adaptée à chaque groupe d'acteurs, sur base d'un classement des habiletés cognitives et sociales à développer par ordre croissant de complexité et de difficulté. 
Critères pour reconnaître qu'un groupe d'acteurs a développé une intelligence commune de son territoire Un soutien politique (existence d'instances de concertation, suivi des projets par des élus et des fonctionnaires, transparence politique, soutien financier, etc.).

Une grande confiance dans les processus participatifs et une compréhension de ces derniers.

Les parties prenantes acceptent l'idée de faire partie d'une communauté d'apprentissage (manifestent le désir et la capacité d'ouverture et de dialogue avec des personnes ayant des points de vue différents, voire opposés).

Le plaisir, la reconnaissance mutuelle, la connivence entre tous et le souci d'intégrer des "gens silencieux $"$.

Un ancrage territorial commun (une connaissance mutualisée des lieux et des gens).

Le souci de la présence des «non humains » sur le territoire. Une mobilisation sur le long terme.

La capacité de changer de statut et de rapports de force entre acteurs. L'existence d'un réseau important et solidaire.

Une distance critique partagée et une éthique commune.

La capacité de chacun de se comporter comme micro-macro-acteur.

L'existence d'un référentiel-qualité du territoire co-élaboré par un grand nombre d'acteurs différents et soumis à la validation de la population.

L'existence d'un document qui traduise une vision commune et durable du territoire.

Une ouverture à l'innovation et à la transversalité de la part des fonctionnaires et des politiques.

Pédagogie et communication : le souci d'assurer la transmission et l'évolution de l'intelligence commune du territoire construite.

Examinons plus en détail ces critères.

Un soutien politique (existence d'instances de concertation, suivi des projets par des élus et des fonctionnaires, transparence politique, soutien financier, etc.)

Les responsables politiques savent comment impliquer dans la durée des acteurs aux intérêts immédiats distincts vers un dessein commun partagé et global (decision framing - préparer la décision -, par opposition à decision making - prendre des décisions). Cela implique de créer des lieux de concertation où les différents protagonistes puissent se réunir, échanger et négocier, avec l'aide d'un intervenant extérieur, ce qui est le gage d'un travail dégagé des pressions politiques ou autres. Cela exige aussi de la part des responsables politiques d'adhérer au principe de la transparence politique, "qui permet de démontrer au citoyen la pertinence de leurs choix pour l'intérêt collectif ou à tout le moins de lui laisser fonder son propre jugement sur la question » (Harou, Fontaine et Rosinski, 2003, p. 36).

Une grande confiance dans les processus participatifs et une compréhension de ces derniers 
39 Les habitants du territoire n'ont pas peur de faire part de leur point de vue aux autorités locales et ont confiance dans le fait que leur parole sera prise en compte. Ils savent où, quand et comment ils peuvent le faire. Les acteurs impliqués dans un processus de participation en connaissent et en comprennent non seulement les résultats, mais aussi les tenants et les aboutissants : en participant, ils acquièrent des notions et des savoir-faire à propos de la participation (Scheers, 2009 : Cockx, 2007 : Santens, Scheers et Struyven, 1983). Les processus participatifs sont internalisés par une «masse critique» d'acteurs, au point de devenir eux-mêmes des vecteurs contribuant à la diffusion de cette nouvelle culture (voir point 15). De même, de la part des acteurs qui ont légitimement un pouvoir de décision ou d'action dans le développement et la gestion des espaces publics, il y a un lâcher prise à accepter et qui constitue un objectif d'apprentissage que l'animateur territorial doit intégrer dans le processus d'accompagnement.

Les parties prenantes acceptent l'idée de faire partie d'une communauté d'apprentissage

41 Alors que l'intention d'apprentissage des acteurs est faible ou absente au début d'un processus participatif, les acteurs acceptent d'apprendre des autres à propos du territoire ou de la manière de le gérer (Wildemeersch et Beckers, 1997), parce qu'ils savent par expérience qu'aucun d'entre eux, aucune institution ne peut, isolément, prétendre être en mesure d'identifier l'ensemble des qualités d'un territoire ou les problèmes qui s'y posent. Ils savent pourquoi il est plus important de travailler à partir des représentations du territoire de chacun pour construire ensemble de nouveaux savoirs, plutôt que de partir de savoirs "savants" élaborés par des experts scientifiques. Ils ont compris que la caractérisation des problèmes avait une valeur relative et que, selon le point de vue des différents acteurs, les problèmes ne sont pas les mêmes, ou pas perçus avec la même acuité (Brédif, 2004), mais ils ont pu constater en même temps que le croisement des regards fait en général apparaitre une problématique territoriale commune que chacun ne percevait pas ou mal, et qui permet de bâtir un socle commun pour travailler ensemble.

Plaisir, reconnaissance mutuelle, connivence entre tous et intégration des "gens silencieux "

Le groupe d'acteurs est uni sur le plan affectif: les personnes ont du plaisir à se retrouver et à œuvrer ensemble, elles se reconnaissent mutuellement comme « interlocuteurs valables ", elles sont capables de plaisanter ensemble et ont développé un humour commun. En outre, le groupe manifeste un réel souci d'accueil et d'intégration des gens habituellement "silencieux», qui se trouvent en général en marge du processus : il accepte que des méthodes spécifiques soient mises en œuvre pour les aider à participer et assurer que ce qui s'exprime puisse être entendu et pris en compte par ceux qui ont le pouvoir de décision.

\section{Un ancrage territorial commun}

Les acteurs témoignent non seulement d'une connaissance et d'une compréhension approfondie du territoire et de son évolution, mais aussi d'une grande sensibilité, d'un attachement fort pour ce territoire. Ils ont construit ensemble une part de cet ancrage affectif et de ces connaissances et disposent d'un vocabulaire commun pour dire le territoire. Cette sensibilité et cette connaissance sont ancrées concrètement sur des relations étroites avec les lieux et les gens. Dès lors, le développement d'une intelligence commune des espaces publics commence sans doute par une étape qui leur 
donne naissance sur le plan symbolique. Le fait de se réunir pour en parler est une manière de les faire exister aux yeux de chacun et leur donne une valeur.

\section{Le souci de la présence des non-humains sur le territoire}

Le groupe manifeste un respect et une compréhension des préoccupations et des revendications des acteurs qui défendent ce que l'on appelle communément «la nature » (Dominique Cottereau, intervention orale à La Rochelle, en 2010). Il évolue progressivement vers la reconnaissance et l'« acceptance » de la relation profonde qui nous lie à notre environnement, dans des formes éminemment différentes d'une culture à l'autre.

\section{La mobilisation à long terme}

Un grand nombre de personnes du territoire manifestent une motivation à son égard, se sentent concernées par son devenir et sont désireuses de s'impliquer concrètement et à long terme, à des degrés divers, ou au moins se tiennent au courant de ce qui se passe. Les fonctionnaires viennent en appui à cette mobilisation et assurent la continuité de mandat en mandat. Les acteurs concernés, dont une large part de la population, adhèrent aux décisions, ou au moins comprennent les motifs qui légitiment les décisions qui sont prises concernant l'aménagement et la gestion du territoire (Harou, Fontaine et Rosinski, 2003), témoignant par là de l'adéquation de ces dernières avec le terrain. Ils sont capables d'intégrer des manifestations de résistance à un projet pour en arriver progressivement, via la concertation et la co-élaboration, à l'acceptation par le plus grand nombre, puis à l'« acceptance» (adhésion aux valeurs qui sous-tendent un projet).

\section{La capacité de changer de statut et de rapports de force entre les acteurs}

Le groupe reconnait l'importance de rompre avec un système fondé sur une coupure entre ceux qui pensent et ceux qui exécutent, entre acteurs (qui décident) et usagers, entre experts scientifiques et experts de terrain, entre «méchants responsables » et "bons citoyens", entre meneurs et suiveurs, entre commanditaire et prestataire de service, etc. Conscient de la difficulté pour chacun de sortir de ses rails, le groupe veille à mettre en place des dispositifs de travail et de communication qui aident à changer de posture. Cette capacité de remettre en question son statut est liée à l'émancipation sociale de chacun, ainsi qu'à l'émancipation du groupe en tant que tel vis-à-vis des acteurs n'en faisant pas partie.

\section{Un réseau important et solidaire}

Les acteurs du territoire, internes et externes, se connaissent bien et sont étroitement reliés entre eux par des liens de solidarité plutôt que des liens de concurrence ou d'antagonisme (Lardon, 2006). Grâce au réseau, ils sont sortis de leur sentiment d'isolement à défendre les qualités du territoire qui leur sont chères, et sentent qu'ensemble, ils sont plus forts. Ils savent cependant qu'il convient d'établir des relations de partenariat très claires, en identifiant bien ce que chacun en attend, ce que chacun peut ou pas apporter, jusqu'où chacun peut s'engager et jusqu'où ne pas aller au-delà au risque de briser les liens ou de modifier la relation partenariale. Le groupe sait aussi reconnaître l'importance de tenir compte des différentes temporalités des uns et des autres ; il est confiant dans sa créativité pour inventer une manière originale de les articuler.

Une distance critique partagée et une éthique commune 
55 Au-delà du « quoi » et du « comment », les acteurs se posent des questions éthiques : ils interrogent le système de valeurs et cherchent une cohérence entre l'être et l'agir en termes d'équité, de justice sociale et d'écojustice : qui décide quoi sur le territoire ? Au nom de qui ? En référence à quelle idéologie? Pourquoi? Le groupe est également capable d'autoréflexivité : qui sommes-nous pour définir un projet de territoire ? Quelle est notre légitimité ? Quels sont nos cadres de référence explicites et implicites ? Le groupe d'acteurs manifeste ainsi de la construction progressive d'une identité politique commune, en explicitant ses représentations du pouvoir et en s'interrogeant sur la place qu'il pense avoir/pouvoir occuper dans la gestion du territoire.

\section{La capacité de chacun de se comporter comme micro-macro-acteur}

Le groupe considère qu'il est important de constituer un groupe d'acteurs qui travaillent à des échelles différentes, que ce soit à l'intérieur ou à l'extérieur du territoire (Guihéneuf, Cauchoix, Barret et Cayre, 2006). Les acteurs sont capables d'être à la fois micro et macro-acteurs, c'est-à-dire être experts sur la thématique traitée, mais aussi de savoir réfléchir à la problématique liée à cette thématique dans le cadre de leur propre vie (Ollagnon, commentaires lors du séminaire Topozym, 2008). Les acteurs locaux ont une bonne connaissance des problèmes qui se posent à l'échelle d'organisation et de responsabilité supérieure et se sentent investis d'un rôle en la matière, tandis que les acteurs à une échelle supérieure ne se cantonnent pas dans des réflexions et des propositions abstraites et théoriques, déconnectées du terrain.

\section{L'existence d'un référentiel-qualité du territoire co-élaboré par un grand nombre} d'acteurs différents et soumis à la validation de la population.

59 Le groupe témoigne, sur base d'une mise en patrimoine commun des qualités du territoire, d'une capacité à travailler ensemble pour mener un diagnostic partagé, pour dégager des problématiques territoriales transversales et des enjeux à long terme. Chaque acteur est capable de se distancier de son propre point de vue pour dresser un portrait global et nuancé des grandes qualités du territoire, en indiquant quels acteurs soutiennent quelles qualités.

\section{L'existence d'un document qui traduise une vision commune et durable du territoire}

Le groupe prend le temps de vérifier l'existence d'un possible espace d'intérêt commun, a contrario d'une recherche d'un hypothétique, voire dangereux intérêt général, ou une recherche d'un hypothétique et utopique consensus. Le groupe est en mesure de faire des propositions d'actions sur la base d'un outil de pilotage stratégique du territoire, permettant de recadrer les problèmes initialement perçus. Il est arrivé à transformer un projet particulier en dessein cohérent de territoire, à co-élaborer une vision commune qui ouvre sur des horizons et des perspectives d'un ordre supérieur à ce que chacun apporte directement, et est capable de décrire le futur désiré en des termes précis. Il est attentif aux changements sur le territoire, et en particulier aux tendances démographiques (mouvements naturels et migratoires, composition de la population) et à l'évolution des écosociosystèmes. Les politiques ont le réflexe de budgéter les projets en fonction de cette vision cohérente du territoire à long terme, en arrivant dans une certaine mesure à se dégager des limites temporelles de leur mandat. Ils comptent sur les fonctionnaires pour assurer une articulation entre projets, pour éviter l'émiettement.

Une ouverture à l'innovation et à la transversalité de la part des fonctionnaires et des responsables politiques 
63 Les responsables politiques, les responsables de grandes institutions et les fonctionnaires manifestent une grande ouverture aux opportunités et à l'idée qu'il existe des marges de gain et des leviers de changement. Ils peuvent imaginer des procédures plus adaptées aux processus de participation et passer ainsi d'une logique de routine à une logique de projet. Ils ont conscience des limites de leur point de vue et ont confiance dans le processus de concertation, voire de cogestion avec l'ensemble des acteurs du territoire.

64 Les situations ont chacune leur originalité propre, liée au contexte et à la personnalité des divers acteurs en présence, l'Être de chaque lieu, les manières de Faire des acteurs en présence et le Devenir du lieu, de ses usagers et de ses usages sont propres à chaque territoire. Appliquer à ces paysages-territoires que sont les espaces publics une méthodologie standardisée, certes sécurisante pour l'animateur territorial, risque donc de mal fonctionner, ou pas de manière optimale.

Pédagogie et communication: le souci d'assurer la transmission et l'évolution de l'intelligence commune du territoire construite

Les acteurs sont conscients de l'importance de transmettre à tout public (à leur entourage, aux nouveaux arrivants, aux visiteurs du territoire, aux générations futures, à des acteurs d'autres territoires) cette intelligence commune du territoire qu'ils ont mis du temps à développer. Ils deviennent ainsi des garants de la «contagion culturelle " localement et des vecteurs de changement pour d'autres territoires. Ils développent à cet effet des outils et des dispositifs " écopédagogiques » permanents et diversifiés, pour informer et former les différents publics visés.

\section{Développer une intelligence commune du territoire}

Il est évident que le développement d'une intelligence commune du territoire ne va pas de soi, d'autant qu'elle ne fait pas à proprement parler des intelligences développées traditionnellement en famille ou à l'école. Le rôle des intervenants éducatifs est crucial à cet égard, pour préparer le terreau : non seulement pour aider chaque personne à oser s'impliquer dans le devenir du territoire, mais aussi pour former à de nouveaux métiers, appartenant au champ de l'animation ou de la médiation territoriale (Guihéneuf et al., 2006).

68 Par ailleurs, l'intelligence commune d'un territoire donné ne peut sans doute s'apprendre que via une approche situationnelle, dans et avec le groupe d'acteurs concernés par ce territoire. Et le recours aux services d'un "écodiplomate » (Bredif, 2004), d'un «animateur communautaire» (Canada), ou encore d'un médiateur territorial (France), apparaît comme déterminant.

[...] l'expérience montrant que, laissés à eux-mêmes, les acteurs ne parviennent pas ou alors très difficilement à inventer ensemble un futur plus désirable, dans lequel chacun peut jouer un rôle constructif, c'est dans un tiers-acteurs extérieur que réside l'espoir d'un changement favorable. (Bredif, 2004, p. 761)

69 Hervé Brédif justifie ainsi l'appellation d'écodiplomate, qui nous paraît correspondre d'assez près à l'approche globale de l'éducation relative à l'environnement :

Diplomate assurément, puisque l'étymologie du mot dit explicitement l'art de construire des accords (...). Le préfixe éco- résume à la fois la volonté de ne pas disjoindre ce qui typiquement relève de considérations distinctes (l'économie/ l'écologie, les humains/les non-humains), mais aussi, et surtout, parce que 
l'étymologie renvoie bien à ce qu'il se donne pour projet : aider les acteurs à coproduire ensemble un habitat désiré, leur vraie maison, sans se restreindre a priori à telle ou telle dimension. (Brédif, 2004, p. 762) qu'individus, pour former un groupe, mais également en tant que groupe vers d'autres groupes, territoires, etc., «reliant des enjeux résonnant à des échelles territoriales croissant de proche en proche jusqu'à embrasser la totalité du globe " (Brédif, ibid.). Il enjoint les acteurs à établir ensemble un référentiel-qualité du territoire, à voir ce qui les relie, ce qu'ils ont en commun, pour imaginer un véritable dessein de territoire dans une vision «service public » qui galvaniserait les actions et les projets des uns et des autres en leur donnant un sens plus considérable et une portée plus générale. commune du territoire nécessite l'intervention d'une personne dotée de compétences de formateur/formatrice de haut niveau pour accompagner les acteurs concernés, que nous considérons comme faisant partie d'une véritable communauté d'apprentissage. Développer une vision « Pour Nous Tous » d'un territoire donné ne se fait pas du jour au lendemain et implique un travail de socialisation en profondeur, mais aussi d'émancipation des uns à l'égard des autres et d'écocipation (appropriation de son environnement) : le cadre environnemental est non seulement pris en compte, mais le but, c'est qu'il devienne pour les participants un véritable territoire d'action. La finalité est donc que les participants s'impliquent, s'engagent par rapport à leurs espaces publics en particulier, et plus largement par rapport à leur environnement. Les compétences à mettre en œuvre méritent qu'un curriculum spécifique de formation soit établi.

\section{BIBLIOGRAPHIE}

Cockx, F. (2007). Leren voor vormingswerk. Concepten voor het faciliteren van leren. Brussel : SoCius.

Brédif, H. (2004). Le vivant, les hommes et le territoire - Essai de biogéopolitique. Thèse de doctorat, Institut National Agronomique Paris-Grignon / École Normale Supérieure Lettres et Sciences humaines Lyon.

Cottereau (2010, juin). Habiter par corps. Communication présentée au Colloque Habiter : l'ancrage territorial comme support pour l'éducation à l'environnement, La Rochelle, France.

De Graef, S., Partoune, C., Schmitz, S., Dalimier, I., Ericx, M. et Scheers, L. (2009). Dashboard aimed at decision-makers and citizens in place management, within SD principles. Final report pour la Politique scientifique fédérale belge. KUL, ULg, IEP, Voormingplus Antwerpen. Consulté le 2 janvier 2010 sur http://www.topozym.be

Fastrez, P. (1999). Aspects sémio-cognitifs de la navigation hypertextuelle - Approche théorique. Mémoire en DEA Communication, Louvain-la-Neuve, UCL.

Gardner, H. (1997). Les formes de l'intelligence. Paris : Odile Jacob. 
Garnier, C. et Sauvé, L. (1999). Apport de la théorie des représentations sociales à l'éducation relative à l'environnement - Conditions pour un design de recherche. Éducation relative à l'environnement - Regards, Recherches, Réflexions, 1, 65-77.

Guihéneuf, P.-Y., Cauchoix, F., Barret, P. et Cayre, P. (coord.) (2006). La formation au dialogue territorial. Dijon : Educagri.

Hansotte, M. (2005). Les intelligences citoyennes. Bruxelles : De Boeck Université.

Harou, R. (coord.), Fontaine, P. et Rosinski, Z. (2003). La participation des citoyens à la vie communale : enjeux et pratiques. Namur : Conférence Permanente du Développement territorial, ministère de la Région wallonne. http://cpdt.wallonie.be/fr/node/131

Lardon, S. (2006). Tisser des liens entre acteurs du territoire. Dans Guihéneuf, P.-Y., Cauchoix, F., Barret, P. et Cayre, P. (coord.), La formation au dialogue territorial (p. 57-60). Dijon : Educagri..

Lakoff, G. et Johnson, M. (1985). Les métaphores dans la vie quotidienne. Paris : Éditions de Minuit. Lazzarotti, O. (2006). Habiter, la condition géographique. Paris : Belin.

Ollagnon, H. (2003). Stratégies pour une gestion patrimoniale de la biodiversité. Bulletin de l'Institut royal des sciences naturelles de Belgique, Biologie, 73-suppl.

Ollagnon, H. (2004). Acteurs et patrimoine dans la gestion de la qualité des milieux naturels. Aménagement et nature, 74.

Ollagnon, H. (2008, 21 novembre). Communication non formelle au Séminaire international Recherche action et approche participative dans l'aménagement et la gestion des lieux publics, Bruxelles, Belgique.

Orellana, I. (2005). L'émergence de la communauté d'apprentissage ou l'acte de recréer des relations dialogiques et dialectiques de transformation du rapport au milieu de vie. Dans Sauvé, L., Orellana, I. et van Steenberghe, É., Éducation et environnement. Un croisement de savoirs. Cahiers scientifiques de l'ACFAS, 104, 67-84.

Partoune, C., Schmitz, S., Dalimier, I., Ericx, M., Scheers, L., Noirhomme, S. et De Graef, S. (2009). Tableau de bord «Participation et espaces publics». Pour un développement et une gestion concertée des espaces publics. Rapport pour la Politique scientifique fédérale belge. KUL, ULg, IEP, Voormingplus Antwerpen. Consulté le 2 janvier 2010 sur http://www.topozym.be

Santens, M., Scheers, L. et Struyven, J. (1983) Participatie, denken en doen, vormingscentrum Intermedium vzw, buurtwerk 't Lampeke vzw. Leuven : CIAUD/ICASD.

Scheers, L. (2009). Perspectives d'éducation/formation. Dans Partoune, C. et alii., Tableau de bord «Participation et espaces publics - Pour un développement et une gestion concertée des espaces publics ». Recherche Topozym pour la Politique scientifique fédérale, partenariat Ulg (UGES), UCL (USEG), Institut d'Éco-pédagogie (IEP), Vorming plus Antwerpen. Consulté le 20 mai 2011 sur http:// www.topozym.be/spip/article.php3?id_article $=68$

Stefanini, O. et Vidal, M. (2001). La gestion patrimoniale - Un outil de formation pour une gestion concertée des territoires. Dijon : Educagri.

Storme (2008, 21 novembre). Communication non formelle au Séminaire international Recherche action et approche participative dans l'aménagement et la gestion des lieux publics, Bruxelles, Belgique.

Waterworth, J. A. (2002). Conscience, action et conception de l'espace virtuel : relier les technologies de l'information, l'esprit et la créativité humaine. In Borillo, M.et Goulette, J.-P. (dir.), Cognition et création, explorations cognitives des processus de conception. Sprimont : Mardaga. 
Wildemeersch, D. et Beckers, F. (1997). Leren is participeren. Vorming, 12(3), 161-171.

\section{NOTES}

1. C'est le courant de la cognition expérientialiste, qui donne priorité à l'expérience physique, comme conditionnant toutes les autres (affective, mentale, culturelle).

\section{RÉSUMÉS}

L'éducation relative à l'environnement est interpellée par la nouvelle gouvernance territoriale qui exige la participation de tous les acteurs. La complexité des habiletés sociales et cognitives requises pour qu'un projet de territoire soit véritablement fondé sur une vision commune de tous les acteurs exige que ces derniers y soient préparés, qu'ils aient développé une «intelligence commune du territoire ». Des critères spécifiques pour reconnaître l'existence de cette forme particulière d'intelligence au sein d'un groupe d'acteurs sont proposés à la discussion dans l'idée de baliser un curriculum de formation pour un nouveau métier: "écodiplomate ", ou « animateur communautaire». Quatre axes peuvent être dégagés pour exprimer les dimensions d'une éducation par et pour le territoire ayant cette forme particulière d'intelligence: axes humaniste, politique, épistémologique et praxique.

Environmental education is concerned about the new territorial governance which requires the participation of all relevant actors. The complexity of social and cognitive skills necessary, so that a territorial project is strongly based on one common vision of all the actors, requires that they are prepared and have developed a " common territorial intelligence ". Specific criteria to recognize this particular form of intelligence within a group of actors are proposed for discussion in order to map the teaching curriculum of a new job: " ecodiplomat ", or " community facilitator ". To express the dimensions of education by and for the territory, four lines of reasoning can be drawn : humanist, political, epistemological and praxis-oriented approaches.

\section{AUTEUR}

\section{CHRISTINE PARTOUNE}

Professeure en didactique de la géographie à l'Université de Liège (Belgique), Christine Partoune dirige le Laboratoire de méthodologie de la géographie. Elle est aussi maître-assistante en géographie à la Haute École Libre Mosane. Elle participe aux recherches en éducation relative à l'environnement menées à l'Institut d'Éco-pédagogie, association sans but lucratif dont elle est actuellement présidente. 